\title{
A new mechanical calibration strategy for U-channel extrusions
}

\author{
Christian Arne Raknes ${ }^{1} \cdot$ Jun $\mathrm{Ma}^{1} \cdot$ Torgeir Welo $^{1} \cdot$ Frode Paulsen ${ }^{2}$
}

Received: 1 April 2020 / Accepted: 30 July 2020 / Published online: 11 August 2020

(C) The Author(s) 2020

\begin{abstract}
The extrusion process of aluminum profiles introduces dimensional variations commonly exceeding the dimensional tolerance requirements for fit-up and assembly when using automated welding processes in the automotive industry. Improving dimensional accuracy of extrusions is challenging from both a technical and economical point of view. However, an alternative technical approach is to introduce fast and simple downstream processes, such as mechanical calibration, to achieve components or products with improved dimensional characteristics. In this paper, a new mechanical calibration strategy is proposed based on the principle of combining transversal stretching and local bending. The strategy is tested on a U-channel extrusion-which is a particularly challenging geometry for extrusions concerning dimensional accuracy. The overall objective is to improve gap opening variations on U-channels compared with as-extruded dimensional capabilities, especially in the case of alloys that are water quenched to obtain high strength and ductility. The study is also intended to serve as basis for future optimization of the mechanical calibration process. An experimental platform and die/tool system was developed, and finite element simulation was used as a research tool to re-produce and better understand the calibration process, thus further analyzing the mechanisms and deformation characteristics important to dimensional accuracy. The experimental results show that the mechanical calibration strategy has good potential to improve the dimensional accuracy of gap openings of U-channels by $90 \%$. The study demonstrates that as-extruded profiles with tolerance limits exceeding the requirements of the final component can effectively be calibrated with the proposed method and hence used in high-volume manufacturing.
\end{abstract}

Keywords Mechanical calibration $\cdot \mathrm{U}$-channel extrusion $\cdot$ Dimensional variability $\cdot$ Springback

\section{Introduction}

As a result of the evolving environmental crises, the demands for reducing air pollution, energy consumption, and $\mathrm{CO}_{2}$ emissions have led to a rapid increase in the use of aluminum alloy applications in the automotive industry. Additionally, the regulatory push for more eco-friendly solutions such as the US fuel economy standards for 2016 has accelerated the process, as analyzed by Cheah and Heywood [1]. Compared with traditional steels, aluminum alloys have become a viable

Christian Arne Raknes

christian.a.raknes@ntnu.no

Jun Ma

jun.ma@ntnu.no

1 Department of Mechanical and Industrial Engineering, Norwegian University of Science and Technology, 7491 Trondheim, Norway

2 Benteler Automotive Raufoss, Grøndalsvegen 2, 2830 Raufoss, Norway option as a result of corrosion resistance, recyclability, good manufacturability, and low mass density. Extruded aluminum profiles allow for flexible cross-sectional designs for long 2D shapes, which can integrate multiple design features as well as optimized weight and stiffness solutions. The typical application areas for extruded profiles are statically and dynamically load-carrying components, such as wheel suspension, crash box assemblies, and windshield frames. Scamans [2] also points out an emerging application potential for extruded aluminum profiles in battery casings for electrical cars. During the last two decades, several authors have studied industrial applications of aluminum in the automotive industry. Miller et al. [3] could report that utilizing aluminum spaceframe for the Audi A8 BIW led to a weight reduction of $40 \%$, whereas the mass savings of up to $50 \%$ could be obtained by applying state-of-the-art design strategies for aluminum [4]. However, Tekkeya et al. [5] emphasize the need for new technologies to meet the automotive manufacturer's goal for lighter vehicles.

Numerous applications of aluminum extrusions have been proven successful, yet dimensional variability is a real challenge for automation of welding and fit-up. Automotive 
components have thus strict requirements related to the dimensional accuracy of the final part geometry. One way to obtain consistent results from the forming processes is by controlling the whole processing route including extrusion, heat treatment, forming, etc. to avoid accumulation of "noise" and parameters that result in increased dimensional variability. However, reducing variations from the production process is a complex task. Identifying important parameters using through-process modeling of extruded Al-Mg-Si alloys has been demonstrated by e.g. [6], but future research is still required on this topic. Reducing dimensional variations due to springback in highvolume manufacturing of automotive parts is particularly important to control dimensional variability $[7,8]$.

The gap opening of extruded U-channel profiles can vary by several millimeters and has proven to be particularly difficult to control according to [9]. In addition to local cross-sectional distortion, dimensional variability in terms of twist, straightness, and thickness variations also contributes to the overall dimensional tolerance window of extruded profiles. As a countermeasure, several calibration methods applied to sheet metal formed U-channel profiles have been proposed in the literature. Chou and Hung [10] explained several Ubending-deep drawing calibration methods for high dimensional accuracy forming, including the so-called arc bottoming and pinching die techniques where the material is set up in the through-thickness direction to reduce springback. Also, the double bending technique for springback reduction was discussed and has been proven feasible on steel U-channels [11]. Vollersten [12] concluded that the accuracy of hydroforming makes this process capable of forming and calibrating parts with excellent final geometry. Electromagnetic calibration of U-channels was presented by Golovashchenko [13]. By contour restraining a straight U-channel and applying electromagnetic current, Golovashchenk obtained stress relief of the part and, thus, decreased springback action. Electromagnetic impulse calibration applied to deep drawn U-channels was demonstrated effectively to minimize springback and correcting sidewall curl defects by Iriondo et al. [14] and was later verified numerically by Cui et al. [15]. Another, relatively new, calibration method is pulsed electrohydraulic springback calibration developed by Golovashchenko et al. [16]. Here, the application of pulsed pressure was used to obtain throughthickness stress which reduced or eliminated in-plane stresses and thereby significantly reduced springback.

There is a growing body of research within the field of calibration, and significant research efforts have been done to improve the dimensional accuracy of U-channels. However, there is still a strong need for innovative, inexpensive calibration methods applicable to complex U-channel aluminum extrusions. The objective of the present work is to propose and demonstrate a new, cost-efficient strategy to improve the gap opening variability of U-channels compared with as-extruded profiles. The work is also intended to provide a basis for future optimization of the mechanical calibration process.

The remainder of the paper is organized as follows: Section 2 presents in detail the proposed mechanical calibration strategy by defining mechanical calibration and discussing the mechanical principles behind the strategy. Section 3 lays the foundation for the experimental work by analyzing as-received material properties and describing the experimental platform as well as tool system employed. Subsequently, the measuring process on critical dimensional characteristics of the sample profiles is presented. Section 4 gives the results and discussion which also includes a reproduction of the experimental results by FEA for through process deformations characteristics. Lastly, Section 5 presents the conclusion and future perspectives of the proposed mechanical calibration strategy.

\section{Mechanical calibration strategy}

Mechanical calibration is intended to be implemented as an additional process step in the press line to reduce variability of semi-finished part geometries. Moreover, mechanical calibration can be defined as the process of forming individual members of the cross-section to known, fixed tool surfaces by relative small-displacement forming. The dimensional accuracy in mechanical calibration can be achieved by reducing the stress gradient through the thickness of individual crosssectional members upon forming and thereby minimizing the springback response. The introduction of local bending upon calibration of a U-channel makes it important to perform the forming under a state of stress that minimizes springback. Accordingly, two strategies can be proposed, i.e., simultaneous local bending and transverse stretching and simultaneous local bending and longitudinal stretching as illustrated in Fig. 1.

The graphs in Fig. 2 are based on bending of a sheet (or lower flange) while simultaneously introducing, respectively, longitudinal or transverse stretching to correct the gap variations. Using a simple analytical interpretation of the mechanisms for the latter, the elastic springback curvature of the bottom flange can be expressed as

$\Delta \kappa^{\mathrm{e}}=\frac{M_{\mathrm{p}}}{E I}$

where $M_{\mathrm{p}}$ is the plastic moment, $E$ is the Young's modulus, and $I$ is the second moment of inertia of one unit sheet width. By applying Navier-Bernoulli bending theory in combination with Ludwig's law, the moment- 
Fig. 1 Principle of two calibration strategies: a transverse stretching and $\mathbf{b}$ longitudinal stretching

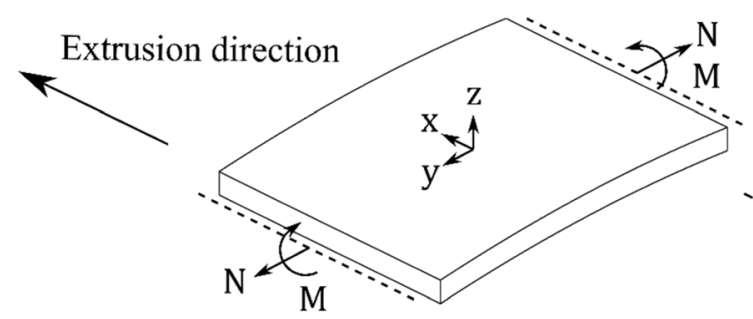

(a)

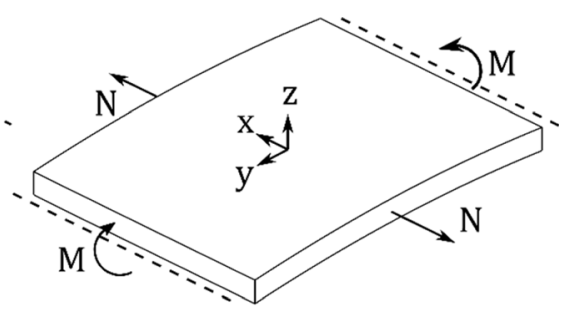

(b) curvature relation for the case of transverse stretching is obtained by solving the following equation for different stretching strains imposed:

$M=\frac{K h^{2}}{\left(\varepsilon_{\mathrm{ey}}-\varepsilon_{\mathrm{iy}}\right)^{2}}\left[\frac{1}{n+2}\left(\varepsilon_{\mathrm{ey}}^{n+2}-\varepsilon_{\mathrm{iy}}^{n+2}\right)-\frac{1}{n+1}\left(\varepsilon_{\mathrm{ey}}^{n+1}-\varepsilon_{\mathrm{iy}}^{n+1}\right) \varepsilon_{\mathrm{my}}\right]$.
This formula applies to the case where the "neutral layer" is located outside the cross-section; hence, the stretching strain is larger than the maximum bending strain at the extremities of the sheet member. $h$ is the flange thickness, and $\varepsilon_{\text {ey }}$ and $\varepsilon_{\text {iy }}$ are the initial strains at the top and bottom of the cross-section, respectively. $\varepsilon_{\mathrm{my}}$ is the applied stretching strain at the mid-depth of
Fig. 2 Mechanical calibration strategies

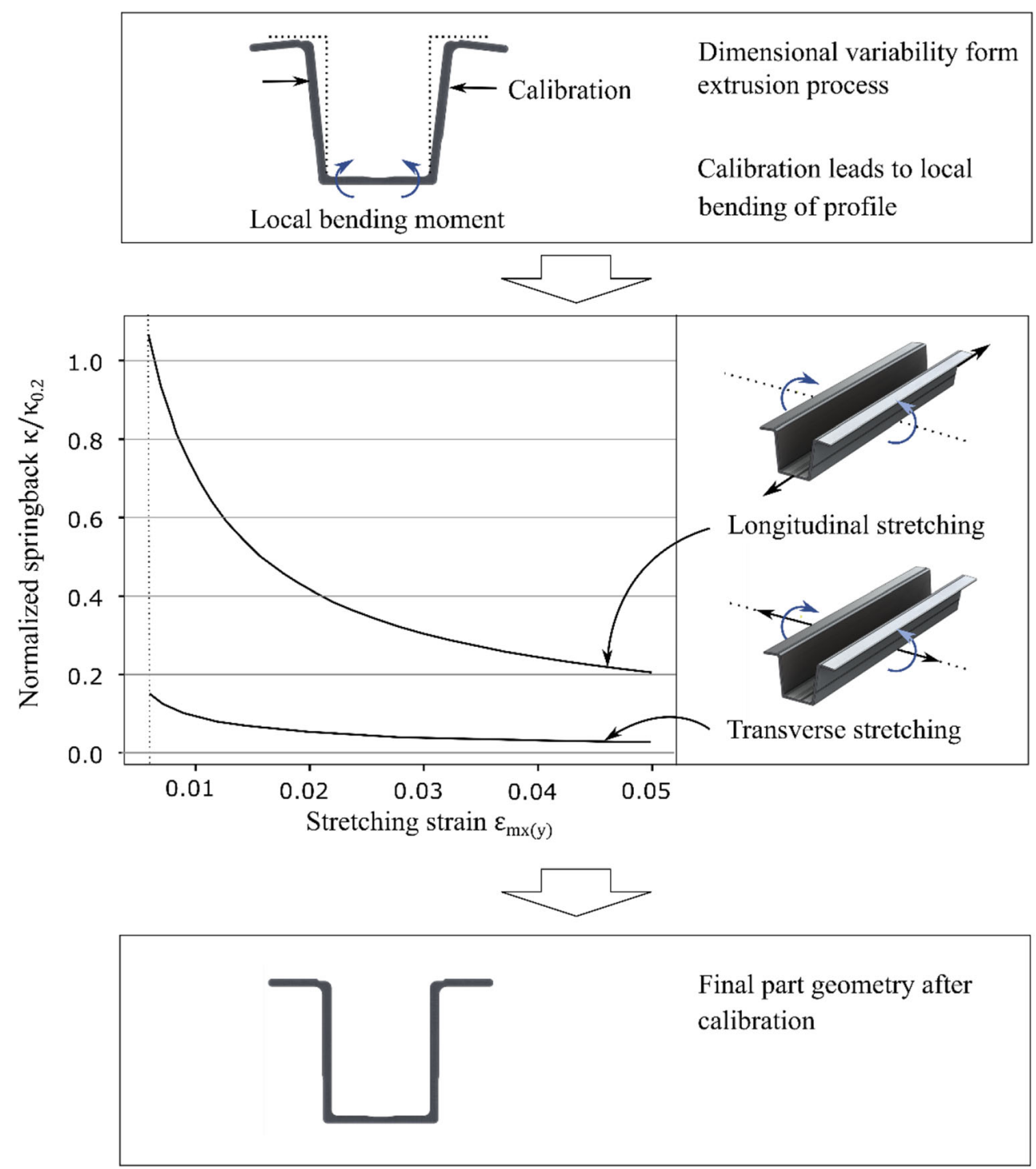


the member. $K$ is the strength factor, and $n$ is the strain hardening parameter in Ludwig's law.

In the other case - longitudinal stretching — an assessment of the longitudinal stretching and local bending can be based on the plastic moment-curvature relationship derived by Welo [17], for suck-in of aluminum extrusions upon global bending:

$M=\frac{\kappa K I}{\left(\varepsilon_{\mathrm{mx}}\right)^{1-n}}$

Where $\varepsilon_{\mathrm{mx}}$ is the applied stretching strain in the extrusion direction and $\kappa$ is the initial curvature of the flange (imperfection). This relationship is only valid when the stretching in the longitudinal direction is dominating; thus, the comparison with the transverse stretching method is only applicable when the neutral layer is outside the cross-section.

The graph in Fig. 2 illustrates the advantage of the transverse stretching strategy over the longitudinal stretching strategy, where the former provides significantly less springback. It is observed that applying longitudinal stretching levels up to above $5 \%$ is necessary to match the transverse stretching strategy at moderate stretching levels, which yields a great risk of contraction effects due to mass conservations. However, the longitudinal stretching still provides a significant reduction of springback compared with pure bending. The transverse stretching strategy provides several advantages as it yields a larger reduction in springback with potentially fewer process steps. Less springback leads to less dimensional variability and therefore better process capability. One aspect associated with the transverse calibration strategy is that the nominal gap opening has to be designed narrower than the intended dimensions to end up at the target width of the profile. Based on the theoretical evaluation above, the transverse stretching strategy is proposed as the most feasible method for mechanical calibration of complex U-shaped aluminum extrusions. To summarize, the proposed mechanical calibration strategy is based on the principle of simultaneously stretching the bottom flange and local bending of the profile, hence superimposing bending and stretching in a nearly one-dimensional state of stress.

The practical approach to achieve the desired stress state is shown in Fig. 3, using a wedge and compound tooling and stretching the initially curved bottom flange, thus inducing local bending until the fixed die surfaces. By moving the wedge, the initial width of the profile is increased by stretching and flattening the initial curvature as illustrated in Fig. 3.

\section{Experiment}

\subsection{As-received profile material}

The U-channel profiles used in this study are made out of a 6082 T4 alloy and were intentionally made outside standard extrusion tolerances with larger-than-nominal gaps. By utilizing these profiles, the mechanical calibration strategy can demonstrate its capability to eliminate geometrical variations and thus also its applicability for extruded profiles with standard tolerances. To determine the specific material properties of the as-received profiles, three tensile tests were conducted. The sample location was placed close to the corner geometry of the profile's bottom flange to capture the most relevant material data for the local bending action. A 3D digital image correlation (DIC) setup was utilized to monitor the strain evolution in both the longitudinal and transverse directions of the specimen. The sampling position in the profile and the geometrical dimension are depicted in Fig. 4. The test sample was designed with a parallel length of $19.34 \mathrm{~mm}, 3-\mathrm{mm}$ thickness, and $5-\mathrm{mm}$ width. The tests were quasi-static with a strain rate of $0.02 \mathrm{~mm} / \mathrm{min}$, and an MTS Criterion Model 42, with a maximum capacity of $5 \mathrm{kN}$, was used for the testing operation. The VIC 3D was employed for postprocessing the image data. Some key material data and chemical composition of the material is given in the engineering stress-strain curve of Fig. 4 and Table 1.

\subsection{Design of experimental platform and tools}

The mechanical calibration setup includes two main parts: a stationary die and a forming tool, as shown in Fig. 5. The stationary die is constructed out of five parts, including a base plate, two solid die blocks, and two profile-specific inserts. The die blocks give the setup sufficient structural integrity, while the profile-specific inserts provide variant flexibility of

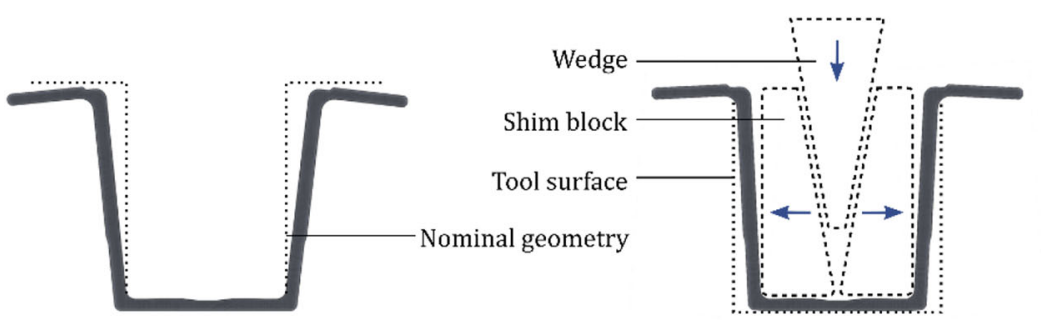

(a) (b)

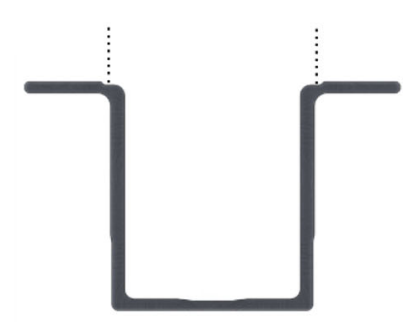

(c)

Fig. 3 Overview of the mechanical calibration process: a as-extruded geometry variations, $\mathbf{b}$ mechanical calibration strategy, and $\mathbf{c}$ calibrated geometry 
Fig. 4 Material property of asreceived AA6082 T4 profile: a Sampling position for tensile testing and $\mathbf{b}$ engineering stressstrain curve



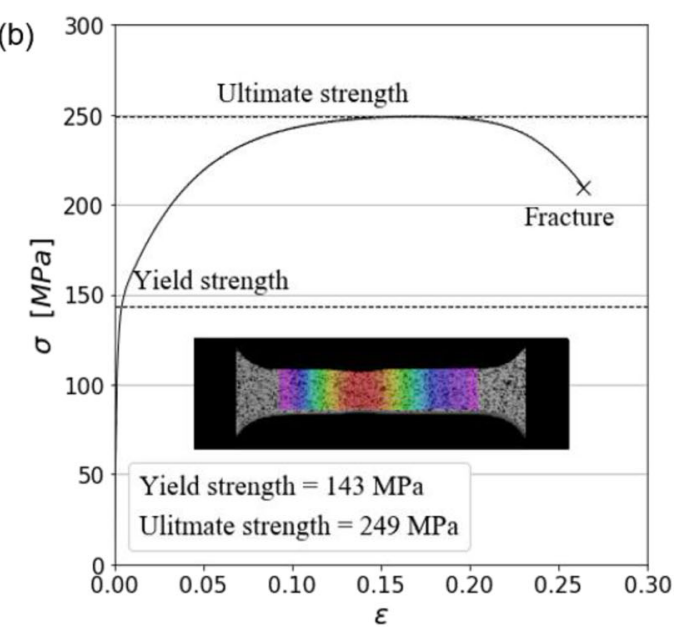

the calibration tool, where the inserts can be altered to fit a variety of profile geometries. To ensure excellent accuracy of the assembly, as well as preventing the die blocks from sliding under loading, ten $\varnothing 10$ stainless steel pins together with ten M16 8.8 bolts were fitted between each die block and base plate. The profile-specific inserts were bolted horizontally with three countersunk M8 8.8 bolts into the die blocks. The forming tool is made up of three parts, consisting of a wedgelike inner unit and two profile-specific shim blocks, designed with a $5.7^{\circ}$ taper and compound which gives $1 / 10$ relation between the vertical wedge displacement and horizontal shim block movement. To ensure proper contact conditions between the shim blocks and profile, the shim blocks were adapted to the internal geometry of the profiles.

The calibration width of the profile was defined based on standard extrusion tolerances of the internal width of the bottom flange, $w=48 \pm 0.6 \mathrm{~mm}$. To ensure at least $2 \%$ plastic strain for the profiles at maximum dimensional tolerance, the bottom flange has to be stretched to $49.67 \mathrm{~mm}$. For comparison, this will give $3.5 \%$ plastic strain for the nominal flange width and $4.8 \%$ plastic strain for a flange with minimum tolerances. This difference will cause some variation in springback, but it was believed that the effect will be low since the natural layer is always outside the crosssection. Finally, by adding wall thickness at the maximum tolerance, the total die opening reaches a width of $58 \mathrm{~mm}$, where the nominal gap opening dimension ends up at $50.2 \mathrm{~mm}$. The test rig, shown in Fig. 6, was reinforced and fitted with two single-acting cylinders to provide the calibration and reversing actions. The wall thickness variations of the profile made a fixed mechanical stop inappropriate for the process, resulting in insufficient calibration at minimum thicknesses and potentially too high force or incomplete movement when the profile was at maximum thickness dimensions. Consequently, it was decided to employ a process that is force-controlled based on the inlet pressure of the downward acting cylinder.

To ensure consistency of the results, a careful experimental procedure was developed. Before each test run, the tool system and profiles were lubricated with Mobil Vactra oil No. 2. The lubrication significantly reduced the effect of friction and the capacity requirements of the hydraulic cylinder. All the profiles were marked at the upper right flange and were placed in the die with the same orientation. The profiles were pressed into the die by a custom steel plate pushed by the wedge. After the profile was properly located, the shim blocks were installed, and the calibration process was initiated by pressurizing the downward-acting hydraulic cylinder. The pressurization continued until the pre-set 180 bar pressure was reached and the release mechanism was activated by the second hydraulic cylinder. Finally, the profile was carefully removed from the die and stored before the last round of measurements.

\subsection{Measurements of profiles}

The 500-mm-long sample profiles (see Fig. 7) were carefully measured following for dimensional tolerances on extruded aluminum profiles. To capture the profile's cross-sectional dimensions along the length of the profile, a coordinate
Table 1 Chemical composition of 6082 alloy [18]

\begin{tabular}{llllllllll}
\hline Element & $\mathrm{Si}$ & $\mathrm{Mg}$ & $\mathrm{Mn}$ & $\mathrm{Fe}$ & $\mathrm{Cr}$ & $\mathrm{Zn}$ & $\mathrm{Ti}$ & $\mathrm{Cu}$ & Others (total) \\
\hline Wt.\% & $0.7-1.3$ & $0.6-1.2$ & $0.4-1.0$ & 0.50 & 0.25 & 0.20 & 0.10 & $0 .-0.1$ & 0.15 \\
\hline
\end{tabular}


Fig. 5 Calibration tool description

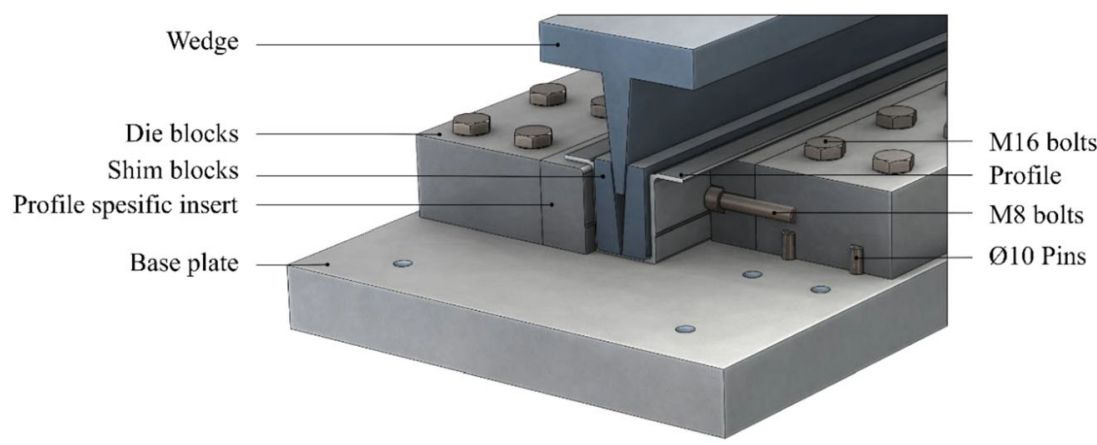

twist, and straightness-were within standard extrusion tolerances.

\section{Results and discussion}

\subsection{Mechanical calibration sequence}

Figure 9 shows different stages of the experimental calibration process in the prototype tool, from initial profile geometry to the finished part geometry. This photograph sequence plainly shows the improvement from the wide gap distortion of the asextruded profile geometry to more parallel U-shaped profiles after calibration and springback.

\subsection{Calibration effect on gap opening}

The most essential calibration effects related to the web-toweb distance variations from the experiment are illustrated in Figs. 10 and 11. Figure 10 presents the profile's end gap in the form of Gap A as previously illustrated in Fig. 8. The first observation from these results is that the maximum variation
Fig. 6 Overview of the calibration testing rig

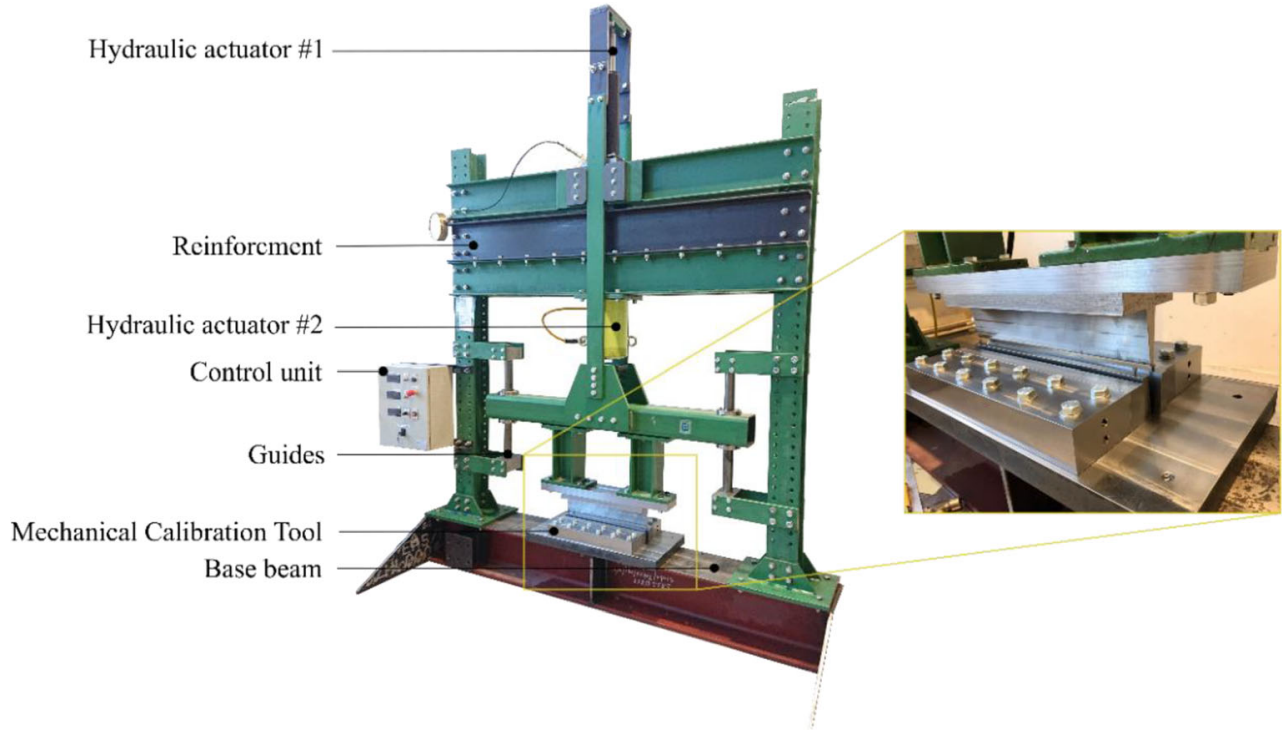




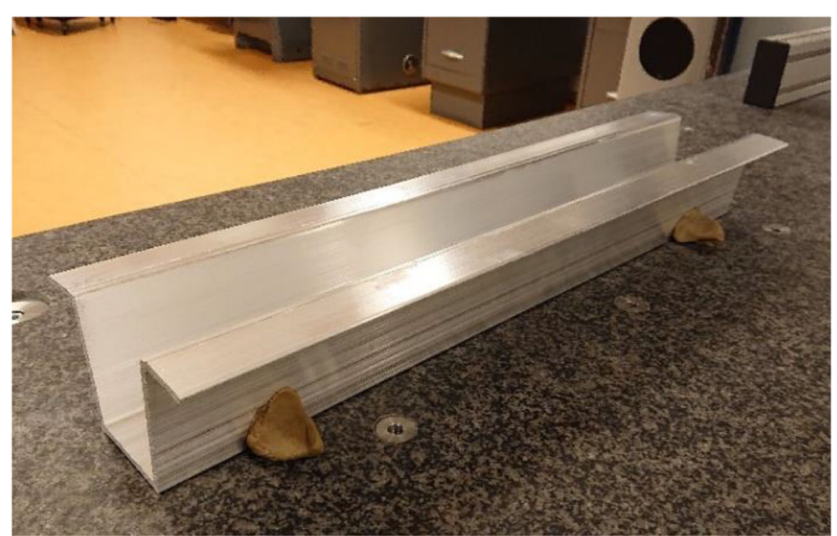

Fig. 7 Overview of profile sample located in coordinate measurement machine

from unformed gap distance to calibrated gap distance is significantly reduced, which is indicated by the nearly flat curve in Fig. 10. From the five samples, the maximum deviations between the widest and narrowest gap are subsequently $4.38 \mathrm{~mm}$ for the as-extruded profiles and $0.42 \mathrm{~mm}$ after calibration. Hence, the maximum gap opening variations are reduced by $90 \%$ after calibration. These findings imply that the mechanical calibration process has a better dimensional capability than what can be achieved directly from the extrusion process, i.e., 0-3-mm gap variations. However, it should be emphasized that the relative improvements obtained from the tested profiles cannot be extrapolated linearly to the capability of the process used for profiles within standard extrusion tolerances. The reason being that the magnitude of variations in springback would be relatively closer to the initial as-extruded gap opening variation and thus less relative improvement is gained.

Based on the nominal wall thickness, the die was designed with a theoretical gap opening of $50.2 \mathrm{~mm}$, whereas the average gap opening after calibration is $51.14 \mathrm{~mm}$. This average gap opening shows that the particular configuration fails to meet the intended nominal gap opening dimensions with a constant off the target dimension close to $1 \mathrm{~mm}$. This is a

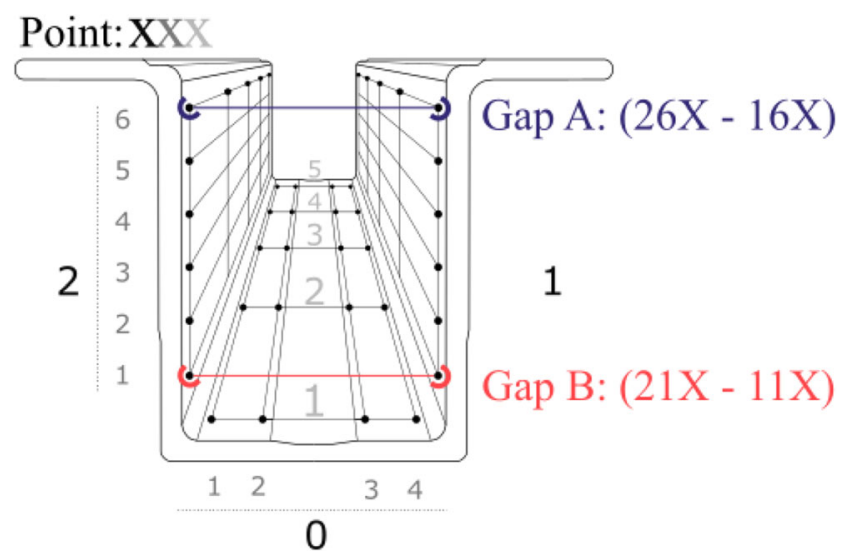

Fig. 8 Point cloud from coordinate measurement machine targeting issue associated with the tool itself and is not necessarily related to the final dimensional variations of the profiles. Figure 11 shows a bar plot of the profile gap opening dimensions ranging from Sections 1 to 5 , where 3 is the longitudinal center of the profile. The error bars are the standard deviation from all of the five samples. One can see that there is a clear trend that the ends, which experience less constraints by the surrounding material, demonstrate a wider gap opening after calibration than the center section along the profile.

\subsection{Calibration effect on profile geometry}

The final geometry of the profile in Fig. 12 shows a bar plot of the five profiles with the average profile gap opening A and the "lower" gap opening B. From this plot, one can see that the process does not produce perfectly parallel webs and that there is a constant offset that makes the profile's webs splay slightly outwards. Considering the initial angularity of the webs, however, the calibration process still significantly improves the "parallelism" of the webs. The error bars show the standard deviations of the through profile gap measures and are related to the through profile springback phenomenon observed in Fig. 11 in the previous section. By illustrating (see Fig. 13) the respective six measurement points from lowest (111) to highest (116) (see Fig. 8) on the right web relative to the lowest point, the overall geometrical shape of the profile web is visualized. The plot shows the change of locations both before and after forming, as well as the reduction of variations. Another observation is that the nominal web shape has gotten a slight curvature. Lastly, Fig. 14 illustrates the outline of a representative profile corner's geometry after calibration, showing that the bottom flange has a slightly concave shape. From the experimental results, it is unclear if the shape originates from the bending action of the bottom flange during calibration or upon the unloading process. This issue will be further analyzed in the FE section.

\subsection{Deformation characteristics identified by FEA}

The physical experiments mainly provide results related to final part geometry and somewhat limited knowledge of the mechanisms throughout the calibration process. To further explore the latter, an FE model was established to gain insight into the deformation characteristics during the whole process, starting from the placement of the profile into the die to final springback, thus providing the basis for the future optimization of the mechanical calibration process.

\subsubsection{FE modeling of mechanical calibration process}

Based on the Abaqus platform, a multistep FE model was established to simulate the mechanical calibration of the Uchannel profile. Figure 15 shows an overview of the FE 

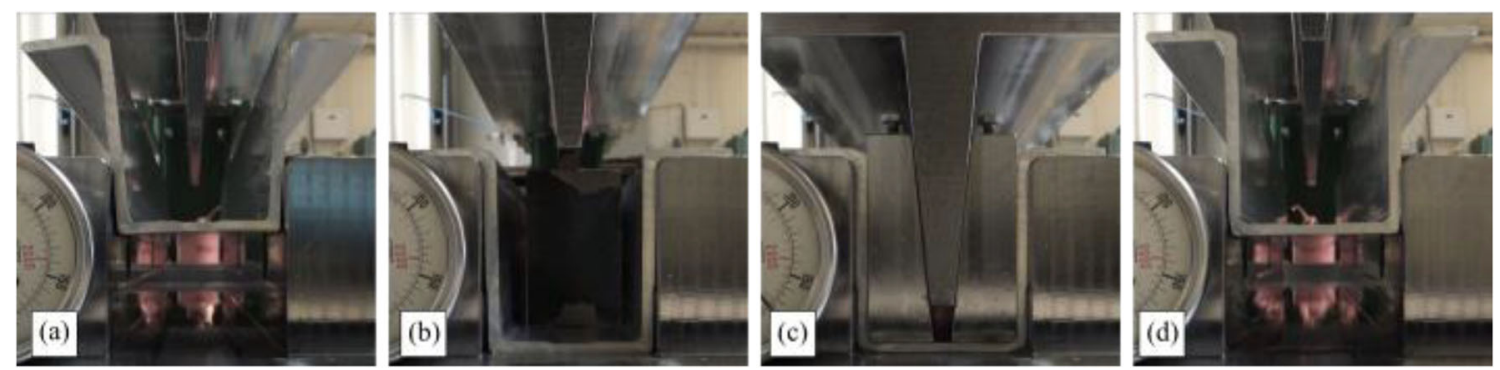

Fig. 9 Mechanical calibration sequence: a initial geometry, b profile placement in die, c calibration, and $\mathbf{d}$ final part geometry

model. The dynamic explicit algorithm was applied to the forming stages, whereas the static implicit algorithm was used for simulation of the springback stages. In total, the simulation was divided into four consecutive stages: placement of profile into the die (insert), springback 1, mechanical calibration, and final springback 2 . The profile and tool system was established as a symmetry model with respect to the xy-plane (see Fig. 15). The geometry dimension of the initial profile is based on the experimentally measured results, in which the gap size uses the average level from experiments. The shim block was given $z$-symmetry conditions at the reference point defined at the mass center. During the springback stages, the $x$-symmetry plan was constrained in the $y$-direction. Also, in the springback 1 stage, the contact point between the profile and die was constrained in the $x$-direction. When simulating the placement of the profile into the die, a vertical velocity of $19.38 \mathrm{~mm} / \mathrm{s}$ was applied to the shim blocks, whereas the wedge was prescribed a vertical velocity of $29.75 \mathrm{~mm} / \mathrm{s}$ throughout the calibration stage. The Coulomb model with a friction coefficient of



Fig. 10 Gap A opening at the front of the profiles $\mu=0.2$ was used to define the contact between profile and tools. Concerning material properties, the Mises yield criterion associated with isotropic hardening law was employed. The material data was extracted from the uniaxial tension test including Young's modulus of $69 \mathrm{GPa}$ and Poisson's ratio of 0.33 . Lastly, the U-channel was meshed with solid linear brick elements, C3D8R, with reduced integration and hourglass control. Rigid elements of the type R3D4 was applied to the tool and die. In the plane zones of the profile, the mesh size was $1 \times 1 \times$ $1 \mathrm{~mm}$ and $0.5 \times 0.5 \times 1 \mathrm{~mm}$ in the corner zones. In the plane zones, the mesh size gives four elements throughthickness which was found sufficient for representing bending problems by Song et al. [19]. A comparison between experiments and simulation of gap A and gap $\mathrm{B}$ is shown in Fig. 16. The results indicate that the relative prediction errors of gap A and gap B are $0.94 \%$ and $0.79 \%$, respectively, showing good prediction accuracy and reliability. Hence, the FE model will be employed to analyze the deformation characteristics of the mechanical calibration process.

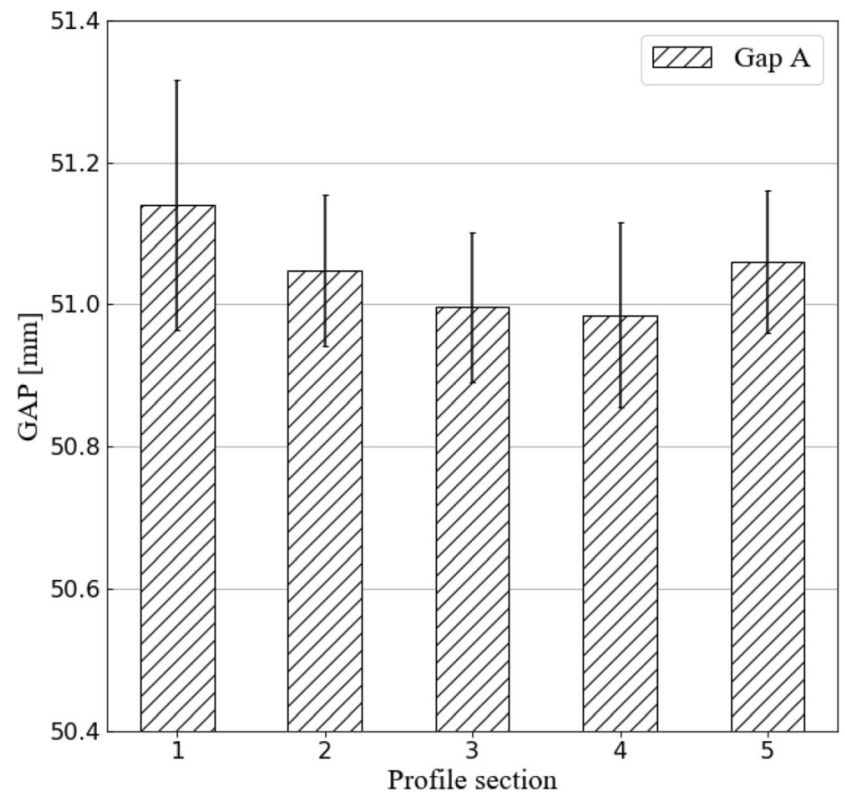

Fig. 11 Average Gap A through profile length 


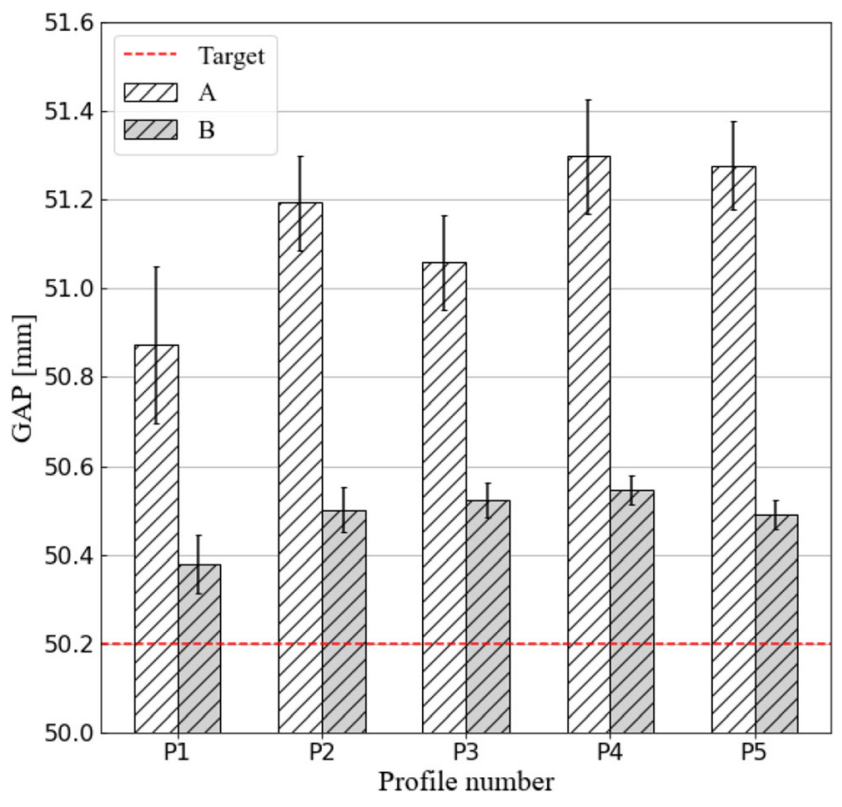

Fig. 12 Gap A and B after calibration

\subsubsection{Analysis of deformation characteristics}

Based on the FE simulation, Fig. 17 shows the overall distribution of effective plastic strain (PEEQ) during the insertspringback-calibration-springback steps. It can be found from Fig. 17a and $b$ that during the insert stage of the as-extruded profile, the plastic strain concentrates in the middle zone of the bottom flange. The maximum effective plastic strain is less than 2\%. As shown in Fig. 17c during the calibration process, the plastic deformation mainly occurs in the corner zone, the sidewall near to the corner, and the middle zone of the bottom flange. The strain concentration is at the inner side of the corner, in which the maximum effective plastic strain can be

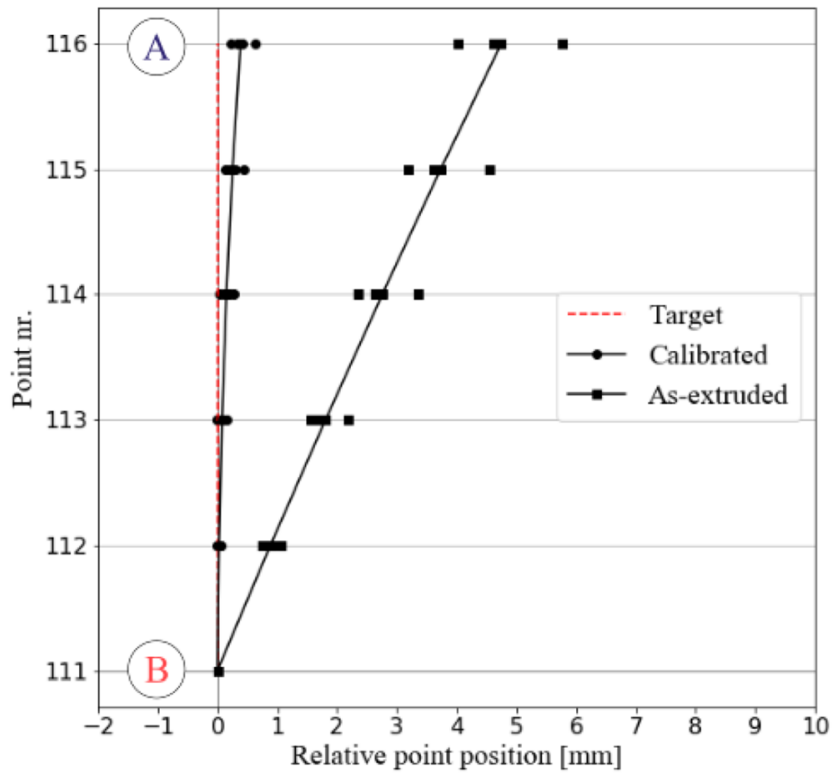

Fig. 13 Right web shape before and after calibration

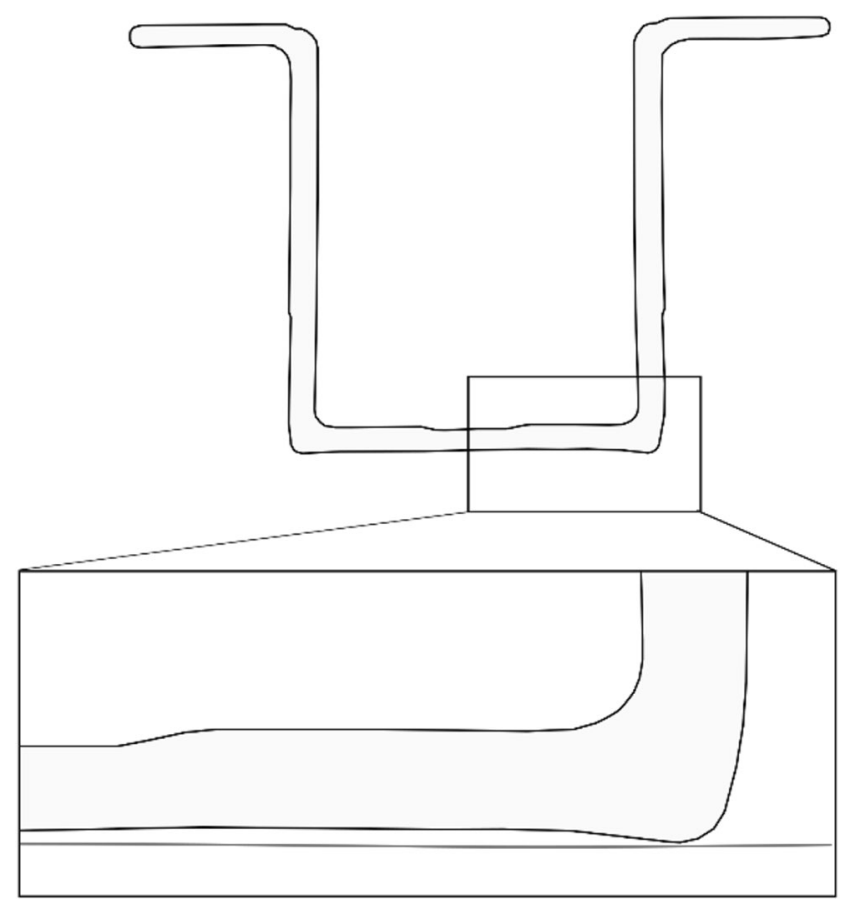

Fig. 14 Outline of local deformation of corner

up to $14 \%$. Hence, we will focus on the calibration stage and the following springback stage to further explore the process behaviors and characteristics.

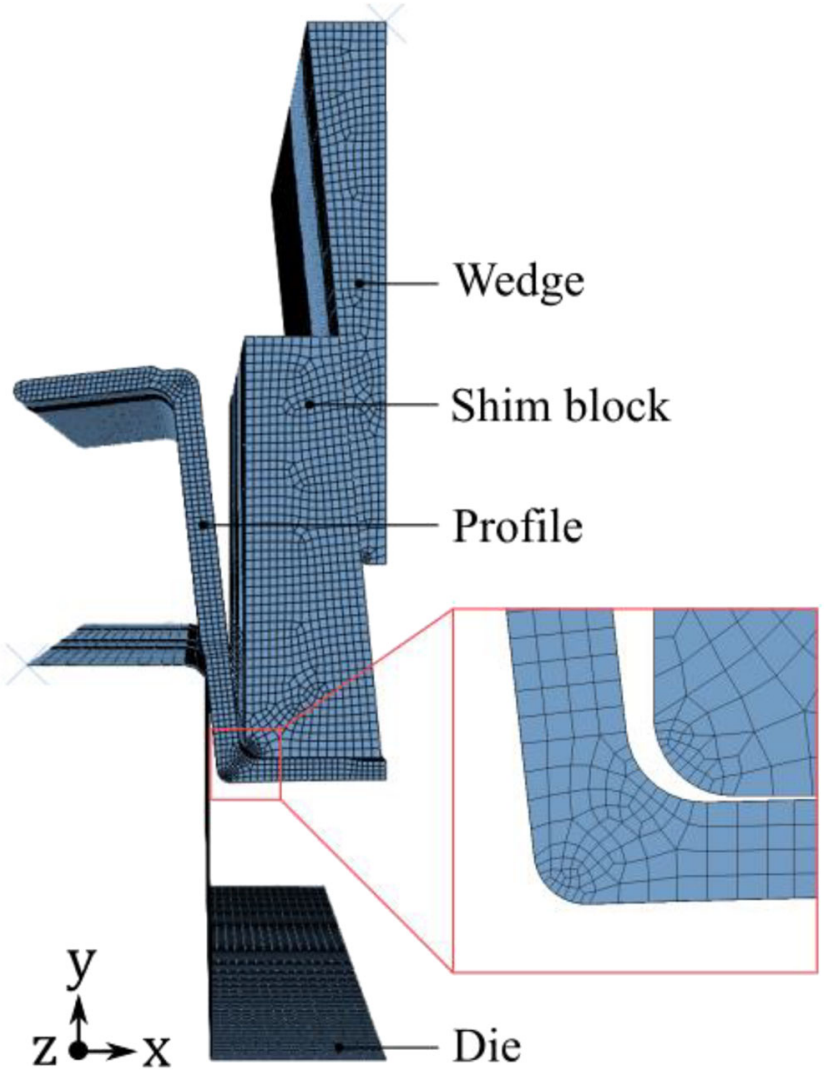

Fig. 15 Overview of FE model 


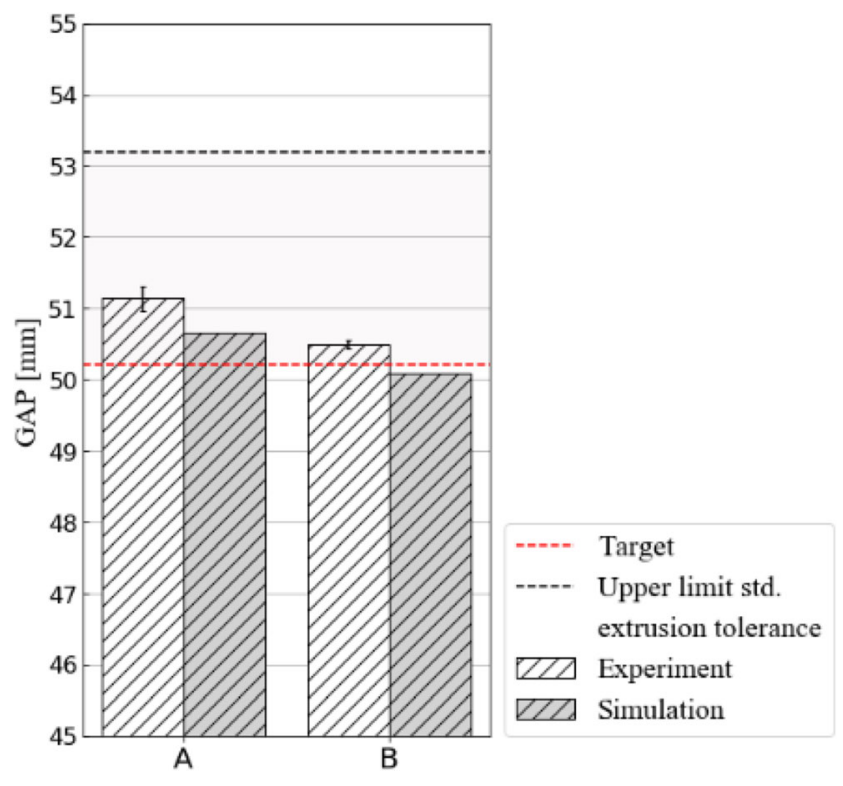

Fig. 16 Comparison between FE simulation and experiment

Figures 18 and 19 show the stress and strain distributions during the calibration process and the final springback process, where S11 and S22 represent the stress components in $x$-direction and $y$-direction, respectively and the PE11 and PE22 mean the strain components in the two directions, respectively. It can be found that the plastic deformation during calibration is mainly focused on the bottom and the sidewall near the bottom corner. In the bottom, tension dominates the deformation during calibration (see Figs. 18a and 19a). Due to the non-uniform thickness, the stress and strain in the thinner middle zone are higher. The tension deformation does not cause stress gradient through the thickness of the bottom flange. However, as can be seen in Fig. 18a or c, some bending of the bottom flange occurs during the calibration stage, hence causing the concave flange in Fig. 14. Since the tension deformation introduces almost uniform stress across thickness, only a slight elastic deformation recovery occurs during the following springback, resulting in limited angular changes in the bottom flange of the profile.

Figure 20 shows the logarithmic strain distribution, in which LE11 and LE22 represent the stress components in $x$-direction and $y$-direction, respectively. It can be found from the corner zone that there is a strain concentration in the interior side of the corner. The strain concentration causes a significant stress gradient in the corner, which creates a large moment in the crosssection of the corner. During springback the moment is partially released, contributing to angular change of the sidewall. For the stress and strain distribution in the sidewall, as shown in Figs. 18c and 19b, there is a significant locally concentrated bending deformation in the sidewall near to the corner zone. From Fig. 19a and, it can be found that the maximum plastic tension strain component PE11 and PE22 at the outer of the local bending zone can be up to -0.04 and 0.04 . After springback, the elastic portion is greatly recovered (see Fig. 20b), which also makes a pronounced difference in the angle of the sidewall.

Based on the multistep simulation as well as the analysis above, it can be concluded that the shape changes during the final springback are mainly caused by the strain concentration of the corner zone and the local bending effect of sidewall near to the corner. To quantitatively characterize the shape change caused by springback, the geometrical dimension of the different points along with the sidewall direction is measured, (a)

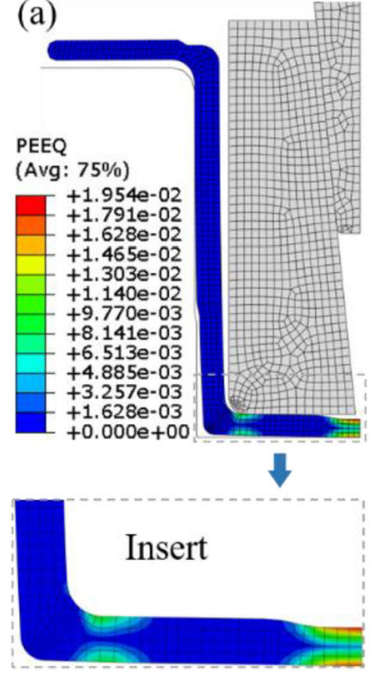

(b)
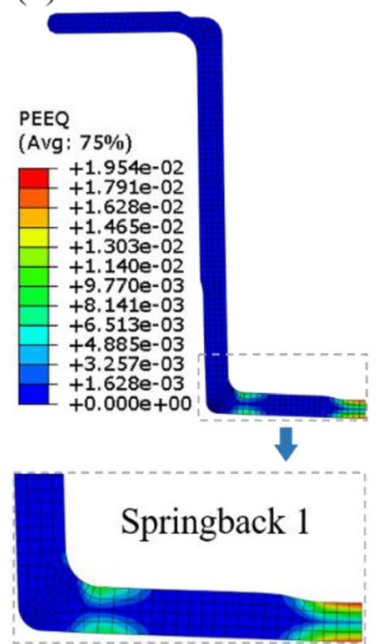

(c)

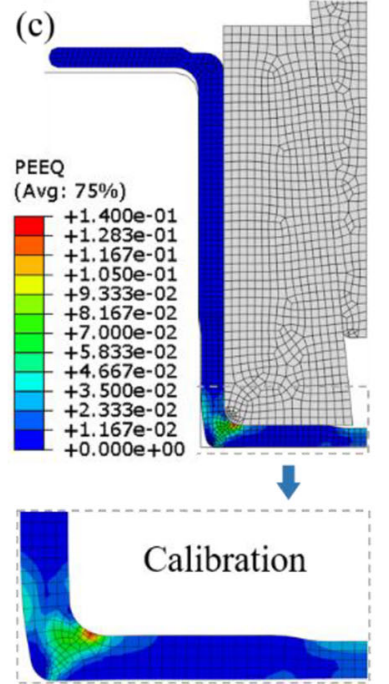

(d)

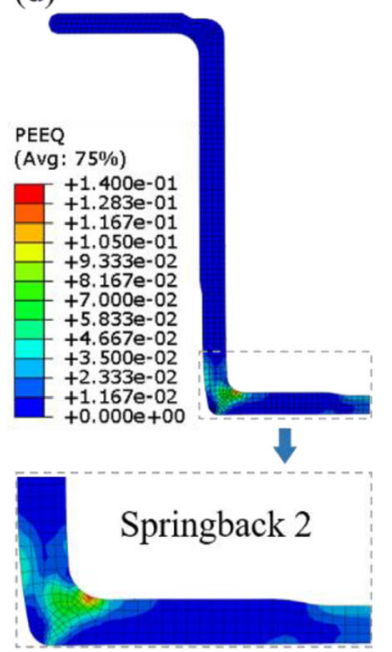

Fig. 17 Simulation of mechanical calibration process: a insert, $\mathbf{b}$ springback, $\mathbf{c}$ calibration, and $\mathbf{d}$ final part after springback 2 


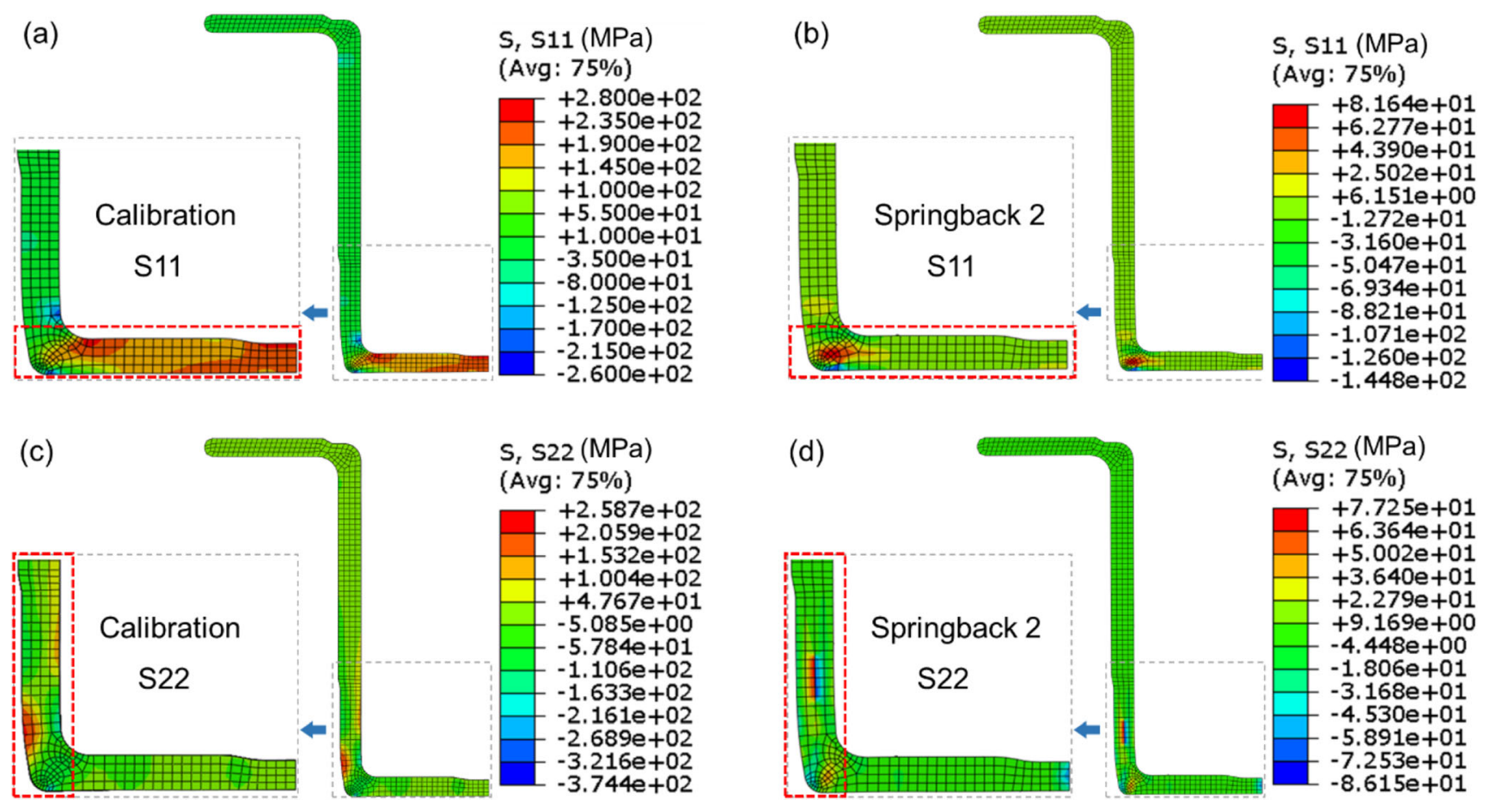

Fig. 18 Stress distribution during mechanical calibration. a Calibration stress S11, b springback stress S11, c calibration stress S22, d springback stress S22

as shown in Fig. 21a and b. Due to springback caused by the strain concentration of corner and local bending in the sidewall, the difference between the target and actual part is increased to $0.39 \mathrm{~mm}$ in the experiment and $0.26 \mathrm{~mm}$ in the simulation. Concerning the shape of the web, the FEA has not quite captured the average experimental web form, i.e., linear vs curved, and needs to be addressed in future research. Even though the variability from springback induced changes is an improvement compared with the variability of standard asextruded profiles, there is still an untapped potential to increase the accuracy of final part geometry. A potential approach is to optimize the tooling design, thus reducing the stress gradient caused by the strain concentration and local bending effect. In sheet metal forming, several methods have been developed to reduce springback; however, in most cases, springback cannot be totally eliminated. Therefore, compensation methods might be explored in combination with the reduction approach to control the final springback in the future optimization.

\section{Conclusions and outlook}

In this paper, a new mechanical calibration strategy for $\mathrm{U}$ channel aluminum extrusion is proposed, tested, and evaluated. The calibration strategy is motivated by the need for improvements of dimensional tolerances for assembly and automated welding in high-volume manufacturing. The main challenges regarding the production of high- (a)

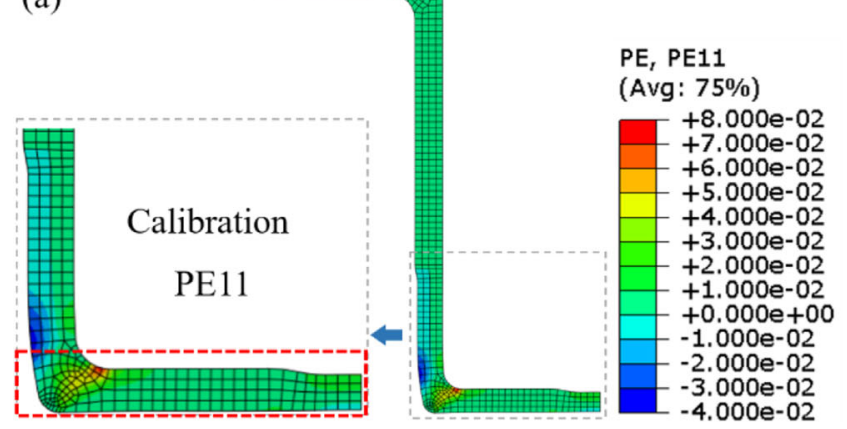

(b)

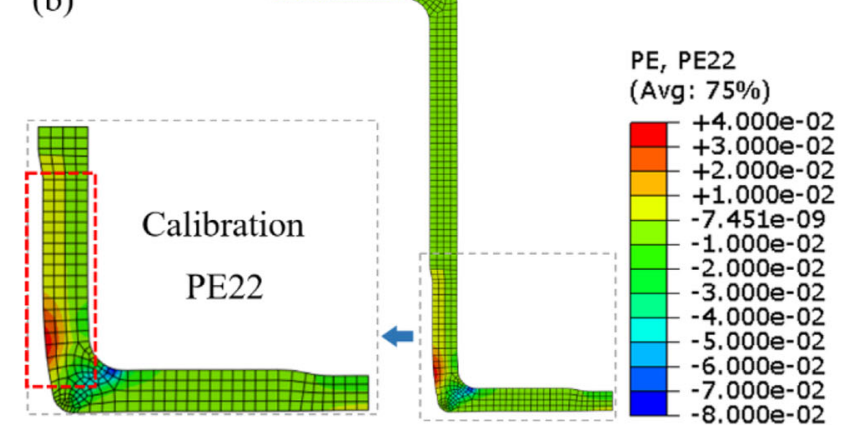

Fig. 19 Strain distribution during final springback. a Calibration plastic strain PE11, b calibration plastic strain PE22 



Fig. 20 Deformation characteristics during springback. a Springback 2 logarithmic strain LE11, b springback 2 logarithmic strain LE22

precision U-channel extrusions are "noise" from the aluminum production chain and the transient nature of the extrusion process itself. Challenges directly related to the mechanical calibration strategy are sensitivity to process parameters, variability of material properties, and part geometry of the incoming extrusions influencing springback. The main conclusions of this study are summarized in the following points:

- The initial hypothesis was that simultaneously introducing transverse stretching in combination with local bending does reduce springback and thereby the dimensional variability. The experimental results showed a $90 \%$ improvement in the maximum gap opening variations for the substandard U-channel profiles. This improvement implies that the process is capable of reducing dimensional variations of asextruded profiles. It also indicates that the process is capable of improving the dimensional variability of extrusions with standard tolerances.
- Based on the multistep FE simulation, the dominating deformation characteristics of the mechanical calibration process were identified as concentrated deformation in the corner region and local bending of the sidewall near the corner. The coupled deformation effect can result in a springback of sidewall after calibration, which implies that further optimization of the process parameters and tool design, in order to obtain an improved state of stress under calibration, would lead to further reduction of springback and thus reduced dimensional variability.

- To reach the next technology readiness level (TRL), future work should consist of research on influencing factors such as material, geometry, and process parameters. This includes FEA accuracy and a statistical capability study on the reduction of dimensional variability.

Funding information Open Access funding provided by NTNU Norwegian University of Science and Technology (incl St. Olavs Hospital - Trondheim University Hospital). The authors would like to acknowledge the financial support from the Norwegian University of Science and Technology (NTNU), NTNU Aluminium Product
Fig. 21 Deformation characteristics during springback. a FE model gap points, $\mathbf{b}$ right web geometry

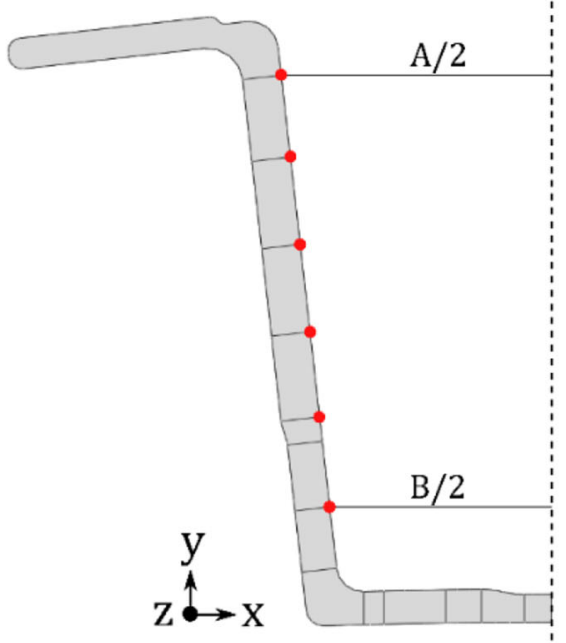

(a)

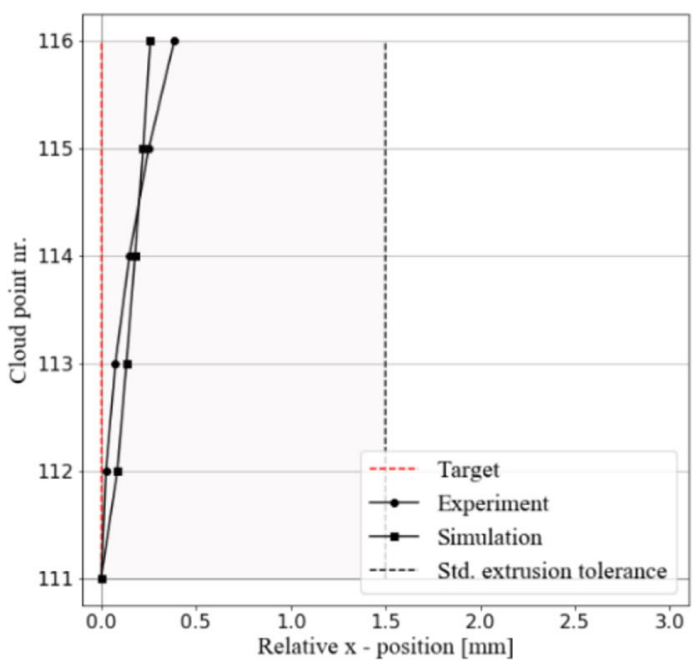

(b) 
Innovation Centre (NAPIC), and the Value Project grant no. 267768 from the Research Council Norway, Hydro and Alcoa.

Open Access This article is licensed under a Creative Commons Attribution 4.0 International License, which permits use, sharing, adaptation, distribution and reproduction in any medium or format, as long as you give appropriate credit to the original author(s) and the source, provide a link to the Creative Commons licence, and indicate if changes were made. The images or other third party material in this article are included in the article's Creative Commons licence, unless indicated otherwise in a credit line to the material. If material is not included in the article's Creative Commons licence and your intended use is not permitted by statutory regulation or exceeds the permitted use, you will need to obtain permission directly from the copyright holder. To view a copy of this licence, visit http://creativecommons.org/licenses/by/4.0/.

\section{References}

1. Cheah L, Heywood J (2011) Meeting U.S. passenger vehicle fuel economy standards in 2016 and beyond. Energy Policy 39:454-466

2. Scamans G (2018) Electric vehicles spike demand for high strength aluminum extrusions. Light Met Age 76:6-12

3. Miller WS, Zhuang L, Bottema J, Wittebrood AJ, De Smet P, Haszler A, Vieregge A (2000) Recent development in aluminium alloys for the automotive industry. Mater Sci Eng A 280:37-49

4. Hirsch J (2014) Recent development in aluminium for automotive applications. Trans Nonferrous Met Soc China 24:1995-2002

5. Tekkaya AE, Khalifa NB, Grzancic G, Hölker R (2014) Forming of lightweight metal components: need for new technologies. Proc Eng 81:28-37

6. Fjeldbo SK, Li Y, Marthinsen K, Furu T (2012) Through-process sensitivity analysis on the effect of process variables on strength in extruded Al-Mg-Si alloys. J Mater Process Technol 212:171-180

7. Ma J, Ha T, Blindheim J, Welo T, Ringen G, Li H (2020) Exploring the influence of pre/post-aging on Springback in Al-Mg-Si alloy tube bending. Proc Manuf 47:774-780
8. Welo T, Ringen G, Ma J (2020) An overview and evaluation of alternative forming processes for complex aluminium products. Proc Manuf 48:82-89

9. NS-EN-755-9 (2016) Aluminium and aluminium alloys - extruded rod/bar, tube and profiles, part 9: profiles, tolerances on dimensions and form. CEN, Standard Norge

10. Chou IN, Hung C (1999) Finite element analysis and optimization on springback reduction. Int J Mach Tools Manuf 39:517-536

11. Liu YC (1984) Springback reduction in U-channels-"double-bend" technique. J Appl Metal Work 3:148-156

12. Vollertsen F (2000) Accuracy in process chains using hydroforming. J Mater Process Technol 103:424-433

13. Golovashchenko $S$ (2006) Electromagnetic forming and joining for automotive applications, 2nd international conference on high speed forming, Mar $20^{\text {th }}-21^{\text {st }}, 2006$, Dortmund, Germany

14. Iriondo E, Gutiérrez MA, González B, Alcaraz JL, Daehn GS (2011) Electromagnetic impulse calibration of high strength sheet metal structures. J Mater Process Technol 211:909-915

15. Cui X, Yu H, Wang Q (2018) Electromagnetic impulse calibration in V-shaped parts. Int J Adv Manuf Technol 97:2959-2968

16. Golovashchenko SF, Gillard AJ, Mamutov AV, Ibrahim R (2014) Pulsed electrohydraulic springback calibration of parts stamped from advanced high strength steel. J Mater Process Technol 214: 2796-2810

17. Welo T (2012) An analytical method for prediction of crosssectional deformations in stretch bending. Key Eng Mater 504: 809-814

18. NS-EN-573-3 (2019) Aluminium and aluminium alloys - chemical composition and form of wrought products, part 3: chemical composition and form of products, Standard Norge

19. Song F, Yang H, Li H, Zhan M, Li GJ (2013) Springback prediction of thick-walled high-strength titanium tube bending. Chin J Aeronaut 26:1336-1345

Publisher's note Springer Nature remains neutral with regard to jurisdictional claims in published maps and institutional affiliations. 